\title{
Readmission and hospital mortality after ICU discharge of critically ill cancer patients
}

\author{
Byeong-Ho Jeong ${ }^{10}{ }^{10}$, Soo Jin $\mathrm{Na}^{2 \oplus}$, Dae-Sang Lee ${ }^{2}$, Chi Ryang Chung ${ }^{1}$, Gee \\ Young Suh ${ }^{1,2}$, Kyeongman Jeon $\mathbb{D}^{1,2 *}$ \\ 1 Division of Pulmonary and Critical Care Medicine, Department of Medicine, Samsung Medical Center, \\ Sungkyunkwan University School of Medicine, Seoul, Republic of Korea, 2 Department of Critical Care \\ Medicine, Samsung Medical Center, Sungkyunkwan University School of Medicine, Seoul, Republic of Korea \\ จ These authors contributed equally to this work. \\ *kjeon@skku.edu
}

\section{Abstract}

\section{Background}

Intensive care unit (ICU) readmission is generally associated with increased hospital stays and increased mortality. However, there are limited data on ICU readmission in critically ill cancer patients.

\section{Method}

Suh GY, Jeon K (2019) Readmission and hospital mortality after ICU discharge of critically ill cancer patients. PLOS ONE 14(1): e0211240. https://doi. org/10.1371/journal.pone.0211240

Editor: Shane Patman, University of Notre Dame Australia, AUSTRALIA

Received: October 12, 2018

Accepted: January 9, 2019

Published: January 24, 2019

Copyright: @ 2019 Jeong et al. This is an open access article distributed under the terms of the Creative Commons Attribution License, which permits unrestricted use, distribution, and reproduction in any medium, provided the original author and source are credited.

Data Availability Statement: All relevant data are within the manuscript.

Funding: This work was supported by a Samsung Medical Center grant (OTA1602901) to KJ. The funders had no role in study design, data collection and analysis, decision to publish, or preparation of the manuscript.

Competing interests: The authors have declared that no competing interests exist.

We conducted a retrospective cohort study based on the prospective registry of all critically ill cancer patients admitted to the oncology medical ICU between January 2012 and December 2013. After excluding patients who were discharged to another hospital or decided to end-of-life care, we divided the enrolled patients into four groups according to the time period from ICU discharge to unexpected events (ICU readmission or ward death) as follows: no (without ICU readmission or death, $n=456$ ), early (within 2 days, $n=42$ ), intermediate (between 2 and 7 days, $n=64$ ), and late event groups (after 7 days of index ICU discharge, $n=129$ ). The independent risk factors associated with ICU readmission or unexpected death after ICU discharge were also analyzed using multinomial logistic regression model.

\section{Results}

There were no differences in the reasons for ICU readmission across the groups. ICU mortality did not differ among the groups, but hospital mortality was significantly higher in the late event group than in the early event group. Mechanical ventilation during ICU stay, tachycardia, decreased mental status, and thrombocytopenia on the day of index ICU discharge increased the risk of early ICU readmission or unexpected ward death, while admission through the emergency room and sepsis and respiratory failure as the reasons for index ICU admission were associated with increased risk of late readmission or unexpected ward death. Interestingly, recent chemotherapy within 4 weeks before index ICU admission was inversely associated with the risk of late readmission or unexpected ward death. 


\section{Conclusion}

In critically ill cancer patients, patient characteristics predicting ICU readmission or unexpected ward death were different according to the time period between index ICU discharge and the events.

\section{Introduction}

The development of diagnostic and therapeutic modalities of cancer and improved clinical outcomes have caused an increase in the inflow of critically ill cancer patients to the intensive care unit (ICU) [1]. Despite recent studies suggesting the benefits of intensive care in cancer patients, it is also true that they still have worse clinical outcomes than patients without cancer $[2,3]$. Efforts have been made to identify the risk factors for ICU admission and mortality in cancer patients to improve their outcomes and to reduce the economic burden; however, there is limited data on unplanned ICU readmission and unexpected ward death after index ICU discharge in cancer patients, which is known to be associated with increased in-hospital mortality, length of hospital stay, and medical cost [4,5].

Previous studies that evaluated ICU readmission and unexpected ward death after ICU discharge have been performed in a general population of critically ill patients, not specifically in cancer patients, and have defined ICU readmission and unexpected ward death as an event that occurred during the entire hospital stay period. However, it is not well known whether all subsequent readmissions and unexpected ward death after ICU discharge have the same characteristics, particularly in cancer patients, who generally have a longer hospital stay [6].

Therefore, this study was conducted to investigate the characteristics and clinical outcomes of cancer patients that were readmitted to the ICU or unexpectedly died on the ward after being discharged alive and to identify patient-related risk factors for predicting ICU readmission or unexpected ward death according to the time period between index ICU discharge and the events.

\section{Materials and methods}

We conducted a retrospective cohort study based on the prospective registry of all critically ill patients with active cancer, defined as diagnosis of cancer, and any treatment for cancer within the previous six-month period or recurrent or metastatic cancer documented by medical record, admitted to the oncology medical ICU of the Samsung Comprehensive Cancer Center of Samsung Medical Center (a 1,979-bed tertiary referral hospital in Seoul, South Korea) between January 2012 and December 2013. The ICU has 14 beds and provides care for approximately 350 critically ill cancer patients per year [7]. The institutional review board of Samsung Medical Center approved this study and waived the requirement for informed consent because of the observational nature of the study.

\section{ICU admission, management, and discharge}

In our hospital, intensivists are involved in decision-making for all admissions to the medical ICU. When the patients on the hospital ward show signs of clinical deterioration, their physician or nursing staff call the medical emergency team, including the intensivists, and then the medical emergency team decides whether to admit to the ICU [7]. Admission from an emergency room (ER) or other hospital is also determined through consultation with intensivists. 
Our ICU is a high-intensity ICU, and dedicated intensivists are responsible for day-to-day patient management. Multidisciplinary teams comprised of ICU physicians, bed-side nurses, nurse practitioners that specialize in respiratory care, clinical pharmacists, and nutritionists routinely assessed all patients admitted to the ICU. An ICU discharge was determined by discussion between the multidisciplinary team and the physician responsible for the patient at the general ward. There were no predefined criteria for admission and discharge, but decisions were made based on generally accepted guidelines [8]. Because our hospital does not have a step-down unit, all patients were discharged from the ICU to the general ward. Verbal and written handover was performed between medical staff members, and the ICU team did not routinely follow-up on the patients discharged from the ICU. Readmission to the ICU was decided in the same way as the index ICU admission.

\section{Study patients}

All consecutive critically ill patients admitted to the oncology medical ICU were prospectively registered from admission. During the study period, a total of 1,125 active cancer patients were admitted to the ICU. We excluded patients who died during index ICU admission $(n=265)$, who were admitted for post-operative observation $(n=86)$, who were discharged to another hospital $(\mathrm{n}=42)$, and who were decided not to readmit to the ICU with limitation of care decision by attending physician $(n=41)$; after exclusions, a total of 691 patients who were discharged alive to the general ward were included in this study.

Data on issues such as unplanned ICU readmission and unexpected death on the ward were collected until the patients were discharged from the ward. However, ICU readmission for scheduled procedures or post-operative observations were not counted as unplanned ICU readmission. If a patient was repeatedly admitted to the ICU during the same hospitalization, we collected the data for the first ICU admission as the index admission and the first ICU readmission as the end-point.

\section{Data collection}

The following baseline characteristics were collected on each patient at index ICU admission: Demographic data and Eastern Cooperative Oncology Group [ECOG] performance status, comorbidities, status of malignancy, source of ICU admission, reason for ICU admission, Simplified Acute Physiology Score 3 [SAPS 3], and Sequential Organ Failure Assessment [SOFA] score. As this study included only patients with malignancies, other definitions associated with cancer status were defined as reported previously [9-11]. The extensiveness of the malignancy was classified according to the extent of the tumor and major organ involvement, as reported previously [9-12]. Extensive disease was defined as metastatic or locally extensive disease in patients with oncologic malignancies [13].

Based on the previous reports on unplanned ICU readmission [14-18], the primary outcome variables in this study were unplanned readmission to the ICU or unexpected death on the ward following ICU discharge. The following data and events were collected at index ICU discharge: organ support treatment during index ICU admission, ICU length of stay (LOS), SOFA score, vital signs, Glasgow coma scale (GCS), arterial blood gases, and laboratory findings. Finally, we collected data associated with status at the time of ICU readmission and final outcome at the time of hospital discharge.

\section{Statistical analyses}

All data are presented as median and interquartile range (IQR) or as the number (percentage) of patients. The data were compared using the Kruskal-Wallis test for continuous variables 
and Pearson's $\chi^{2}$ test or Fisher's exact test for categorical variables. If there were multiple comparisons, we corrected the $P$ value using the Bonferroni method.

We used multinomial logistic regression analysis to identify the independent variables associated with events (unplanned ICU readmission or unexpected death at the ward) depending on the time since index ICU discharge (defined as early, intermediate, and late event groups), with the no event group as the reference category. Multinomial logistic regression analysis was performed with backward stepwise selection with a $P<0.05$ for entry of variables and $P>0.10$ for removal of variables. Initial candidate variables were baseline characteristics at index ICU admission and data at index ICU discharge, except variables having missing data. Continuous variables were entered after converting to categorical variables to facilitate clinical interpretation. Results are presented as odd ratios (ORs) with 95\% confidence intervals (CIs). All the tests were two-tailed, and a $P$ value $<0.05$ was considered significant. The data were analyzed using PASW Statistics 18 (SPSS Inc., Chicago, IL, USA).

\section{Results}

Patient distribution after being discharged alive from the intensive care unit (ICU) is shown in Fig 1.
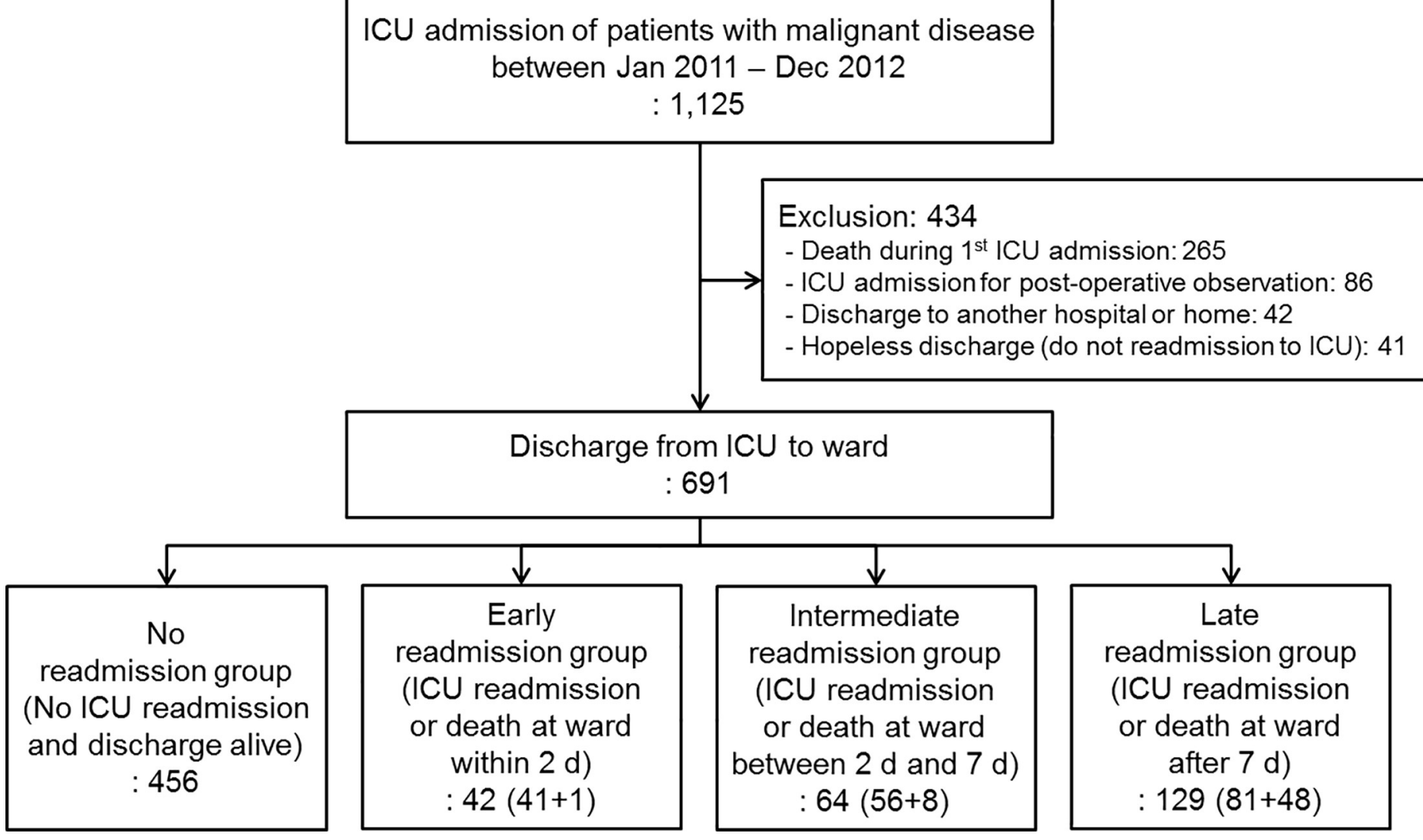

Fig 1. Patient distribution after being discharged alive from the intensive care unit (ICU). A total of 691 patients who were discharged alive from the medical ICU were divided into four groups: No event group, patients who were alive and discharged from hospital without ICU readmission; early event group, patients who were unexpectedly readmitted to the ICU or died on the ward within 2 days after index ICU discharge; intermediate event group, patients who were unexpectedly readmitted to the ICU or died on the ward between 2 and 7 days after index ICU discharge; late event group, patients who were unexpectedly readmitted to the ICU or died on the ward after 7 days of index ICU discharge.

https://doi.org/10.1371/journal.pone.0211240.g001 


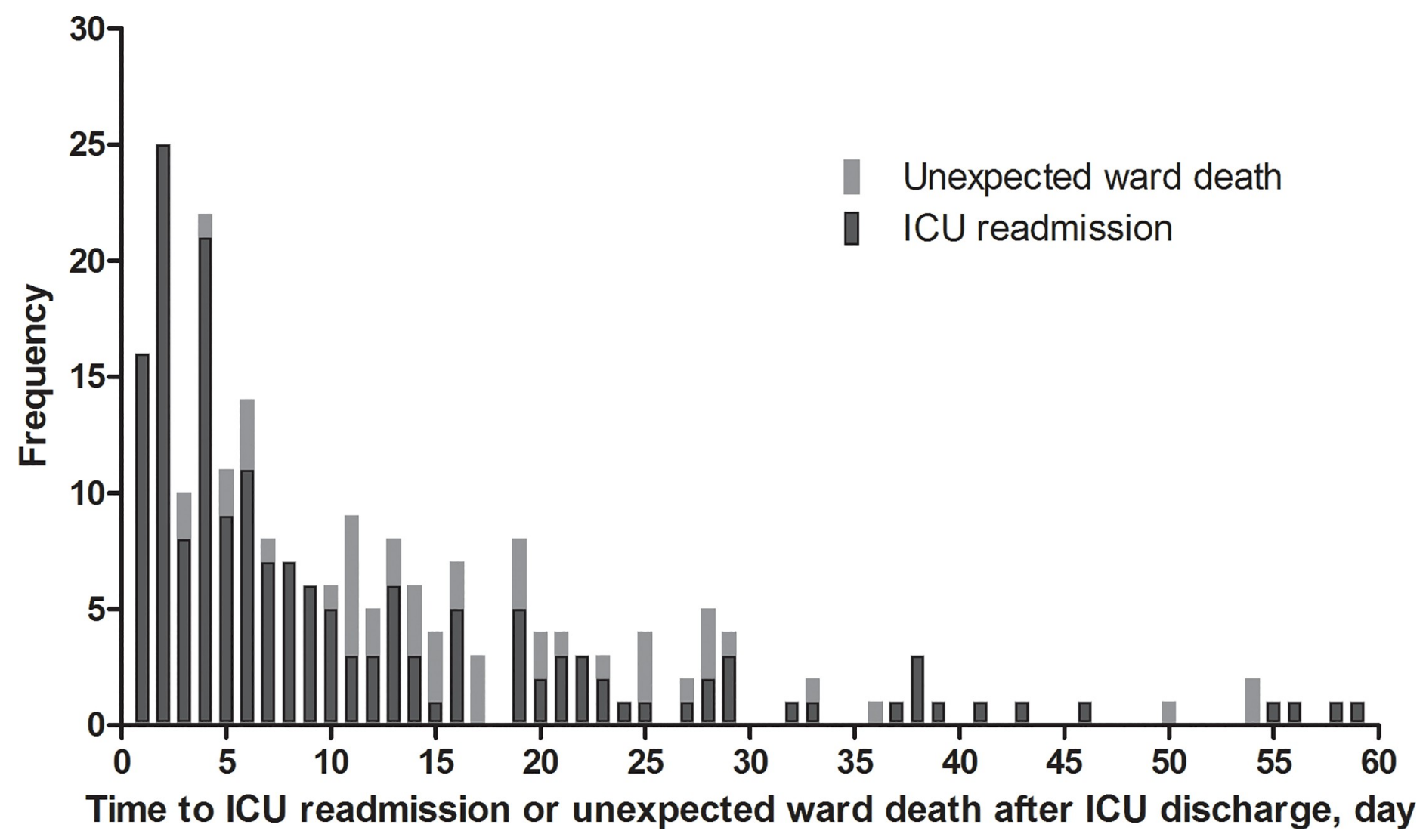

Fig 2. Intensive care unit (ICU) readmission or unexpected death on the ward after being discharged alive from the ICU.

https://doi.org/10.1371/journal.pone.0211240.g002

We divided the 691 enrolled patients into four groups as follows (Fig 1): (A) Patients who were discharged alive from the hospital without ICU readmission (no event group; $n=456$, $66 \%$ ), (B) patients who were unexpectedly readmitted to the ICU or died at the ward within 2 days after index ICU discharge (early event group; $n=42,6 \%$ ), (C) patients who were unexpectedly readmitted to the ICU or died at the ward between 2 and 7 days after index ICU discharge (intermediate event group; $n=64,9 \%$ ), and (D) patients who were unexpectedly readmitted to the ICU or died at the ward after 7 days of index ICU discharge (late event group; $\mathrm{n}=129,19 \%)$.

The number of ICU readmissions and unexpected ward death after index ICU discharge is shown in Fig 2.

\section{Baseline characteristics}

Baseline characteristics and clinical course of index ICU admission are shown in Table 1. There were 243 (35.2\%) patients with hematologic malignancy, and this was more common in the early (50.0\%), intermediate (45.3\%), and late event groups (44.2\%) than in the no event group $(29.8 \%, P=0.001)$. In the late event group, respiratory failure as the reason for index ICU admission was more common (39.5\% vs. $25.4 \%, P=0.010)$, whereas cardiovascular problems were less frequent $(11.6 \% v s .25 .7 \%, P=0.001)$ than in the no event group. SAPS 3 and SOFA scores were higher in the early and late event groups than in the no event group. Mechanical ventilation during index ICU admission was more frequently required in the early (61.9\%), intermediate (46.9\%), and late event groups (45.7\%) than in the no event group 
Table 1. Baseline characteristics and clinical course of index ICU admission.

\begin{tabular}{|c|c|c|c|c|c|c|}
\hline Variables & $\begin{array}{l}\text { Total } \\
(\mathrm{N}=691)\end{array}$ & $\begin{array}{l}\text { No event group* } \\
(\mathrm{n}=456)\end{array}$ & $\begin{array}{l}\text { Early event group } \\
(\mathrm{n}=42)\end{array}$ & $\begin{array}{l}\text { Intermediate event group* } \\
(\mathrm{n}=64)\end{array}$ & $\begin{array}{l}\text { Late event group* } \\
(\mathrm{n}=129)\end{array}$ & $P$ \\
\hline Age, yr & $62(51-70)$ & $63(51-70)$ & $62(51-72)$ & $63(53-68)$ & $62(50-72)$ & 0.997 \\
\hline Sex, male & $456(66.0)$ & $300(65.8)$ & $27(64.3)$ & $52(81.3)$ & $77(59.7)$ & $0.029^{\mathrm{b}}$ \\
\hline $\mathrm{ECOG} \geq 3$ & $97(14.0)$ & $47(10.3)$ & $11(26.2)$ & $11(17.2)$ & $28(21.7)$ & $0.001^{\mathrm{a}, \mathrm{c}}$ \\
\hline Body mass index, $\mathrm{kg} / \mathrm{m}^{2}$ & $\begin{array}{l}21.4(19.3- \\
23.7)\end{array}$ & $21.6(19.4-24.0)$ & $20.5(18.9-23.8)$ & $22.1(20.1-24.0)$ & $20.8(18.7-23.0)$ & 0.104 \\
\hline \multicolumn{7}{|l|}{ Comorbidity } \\
\hline Diabetes mellitus & $157(22.7)$ & $98(21.5)$ & $11(26.2)$ & $16(25.0)$ & $32(24.8)$ & 0.756 \\
\hline Chronic heart disease & $82(11.9)$ & $48(10.5)$ & $6(14.3)$ & $3(4.7)$ & $25(19.4)$ & $0.012^{c}$ \\
\hline Chronic liver disease & $62(9.0)$ & $46(10.1)$ & $2(4.8)$ & $2(3.1)$ & $12(9.3)$ & 0.248 \\
\hline Cerebrovascular disease & $54(7.8)$ & $31(6.8)$ & $4(9.5)$ & $6(9.4)$ & $13(10.1)$ & 0.486 \\
\hline Chronic kidney disease & $52(7.5)$ & $31(6.8)$ & $5(11.9)$ & $5(7.8)$ & $11(8.5)$ & 0.550 \\
\hline COPD/asthma & $34(4.9)$ & $16(3.5)$ & $4(9.5)$ & $4(6.3)$ & $10(7.8)$ & 0.063 \\
\hline \multicolumn{7}{|l|}{ Status of malignancy } \\
\hline Oncologic malignancy & $448(64.8)$ & $320(70.2)$ & $21(50.0)$ & $35(54.7)$ & $72(55.8)$ & $0.001^{\mathrm{a}, \mathrm{b}, \mathrm{c}}$ \\
\hline Extensive disease & $277 / 448(61.8)$ & $192 / 320(60.0)$ & $15 / 21(71.4)$ & $23 / 35(65.7)$ & $47 / 72(65.3)$ & 0.602 \\
\hline Hematologic malignancy & $243(35.2)$ & $136(29.8)$ & $21(50.0)$ & $29(45.3)$ & $57(44.2)$ & $0.001^{\mathrm{a}, \mathrm{b}, \mathrm{c}}$ \\
\hline \begin{tabular}{|l|} 
Stem cell \\
transplantation
\end{tabular} & $54 / 243(22.2)$ & $28 / 136(20.6)$ & 6/21 (28.6) & $5 / 29(17.2)$ & $15 / 57(26.3)$ & 0.629 \\
\hline $\begin{array}{l}\text { First presentation of } \\
\text { malignancy }\end{array}$ & $142(20.5)$ & $88(19.3)$ & $9(21.4)$ & $18(28.1)$ & $27(20.9)$ & 0.437 \\
\hline $\begin{array}{l}\text { Chemotherapy within } 4 \\
\text { weeks }\end{array}$ & $319(46.2)$ & $218(47.8)$ & $22(52.4)$ & $31(48.4)$ & $48(37.2)$ & 0.142 \\
\hline $\begin{array}{l}\text { Source of index ICU } \\
\text { admission }\end{array}$ & & & & & & $<0.001^{\mathrm{ab}, \mathrm{c}}$ \\
\hline Emergency department & $338(48.9)$ & $256(56.1)$ & $19(45.2)$ & $22(34.4)$ & $41(31.8)$ & \\
\hline Others $^{\dagger}$ & $353(51.1)$ & $200(43.9)$ & $23(54.8)$ & $42(65.6)$ & $88(68.2)$ & \\
\hline \multicolumn{7}{|l|}{$\begin{array}{l}\text { Reason for index ICU } \\
\text { admission }\end{array}$} \\
\hline Sepsis & $240(34.7)$ & $150(32.9)$ & $19(45.2)$ & $19(29.7)$ & $52(40.3)$ & 0.157 \\
\hline Respiratory failure & $201(29.1)$ & $116(25.4)$ & $11(26.2)$ & $23(35.9)$ & $51(39.5)$ & $0.010^{c}$ \\
\hline Cardiovascular & $147(21.3)$ & $117(25.7)$ & $7(16.7)$ & $8(12.5)$ & $15(11.6)$ & $0.001^{c}$ \\
\hline Bleeding & $43(6.2)$ & $31(6.8)$ & $1(2.4)$ & $6(9.4)$ & $5(3.9)$ & 0.343 \\
\hline Neurologic disorder & $19(2.7)$ & $12(2.6)$ & $1(2.4)$ & $3(4.7)$ & $3(2.3)$ & 0.733 \\
\hline Others & $41(5.9)$ & $30(6.6)$ & $3(7.1)$ & $5(7.8)$ & $3(2.3)$ & 0.202 \\
\hline $\begin{array}{l}\text { SAPS III at index ICU } \\
\text { admission }\end{array}$ & $49(40-60)$ & $47(37-56)$ & $61(48-71)$ & $53(40-66)$ & $54(44-64)$ & $<0.001^{\mathrm{a}, \mathrm{c}}$ \\
\hline $\begin{array}{l}\text { SOFA score at index ICU } \\
\text { admission }\end{array}$ & $5(3-8)$ & $5(2-8)$ & $8(6-10)$ & $6(3-9)$ & $6(4-9)$ & $<0.001^{\mathrm{a}, \mathrm{c}}$ \\
\hline \multicolumn{7}{|l|}{$\begin{array}{l}\text { Organ support during index } \\
\text { ICU admission }\end{array}$} \\
\hline Vasopressor & $310(44.9)$ & $193(42.3)$ & $25(59.5)$ & $26(40.6)$ & $66(51.2)$ & 0.061 \\
\hline Mechanical ventilator & $256(37.0)$ & $141(30.9)$ & $26(61.9)$ & $30(46.9)$ & $59(45.7)$ & $<0.001^{\mathrm{a}, \mathrm{b}, \mathrm{c}}$ \\
\hline $\begin{array}{l}\text { Renal replacement } \\
\text { therapy }\end{array}$ & $64(9.3)$ & $35(7.7)$ & $5(11.9)$ & $8(12.5)$ & $16(12.4)$ & 0.203 \\
\hline $\begin{array}{l}\text { LOS of index ICU } \\
\text { admission }\end{array}$ & $3(2-6)$ & $3(2-5)$ & $5(2-10)$ & $4(2-8)$ & $4(2-8)$ & $<0.001^{\mathrm{a}, \mathrm{b}, \mathrm{c}}$ \\
\hline $\begin{array}{l}\text { SOFA at index ICU } \\
\text { discharge }\end{array}$ & $3(1-6)$ & $3(1-5)$ & $5(4-7)$ & $4(1-6)$ & $4(2-6)$ & $<0.001^{\mathrm{a}, \mathrm{c}}$ \\
\hline
\end{tabular}


Table 1. (Continued)

\begin{tabular}{|c|c|c|c|c|c|c|}
\hline Variables & $\begin{array}{l}\text { Total } \\
(\mathrm{N}=691)\end{array}$ & $\begin{array}{l}\text { No event group* } \\
(\mathrm{n}=456)\end{array}$ & $\begin{array}{l}\text { Early event group* } \\
(\mathrm{n}=42)\end{array}$ & $\begin{array}{l}\text { Intermediate event group } \\
(\mathbf{n}=64)\end{array}$ & $\begin{array}{l}\text { Late event group* } \\
(\mathrm{n}=129)\end{array}$ & $P$ \\
\hline $\begin{array}{l}\text { Difference value of SOFA } \\
\text { between admission and } \\
\text { discharge }\end{array}$ & $-2(-4$ to 0$)$ & $-1(-3$ to 0$)$ & $-2(-4$ to 0$)$ & $-2(-4$ to 0$)$ & $-2(-4$ to -1$)$ & 0.540 \\
\hline
\end{tabular}

Values are median with interquartile range or $\mathrm{n}(\%)$.

*No event group means patients who were discharged alive from the hospital without ICU readmission. Early event group means patients who were unexpectedly readmitted to the ICU or died at the ward within 2 days after index ICU discharge. Intermediate event group means patients who were unexpectedly readmitted to the ICU or died at the ward between 2 and 7 days after index ICU discharge. Late event group means patients who were unexpectedly readmitted to the ICU or died at the ward after 7 days of index ICU discharge.

${ }^{\text {a }} P<0.05$ with Bonferroni correction between no event and early event groups.

${ }^{\mathrm{b}} P<0.05$ with Bonferroni correction between no event and intermediate event groups.

${ }^{c} P<0.05$ with Bonferroni correction between no event and late event groups.

${ }^{\dagger}$ Transfer from a ward or outside hospital.

ECOG, Eastern Cooperative Oncology Group; COPD, chronic obstructive pulmonary disease; LOS, length of stay; ICU, intensive care unit; SAPS, Simplified Acute Physiology Score; SOFA, Sequential Organ Failure Assessment.

https://doi.org/10.1371/journal.pone.0211240.t001

(30.9\%, $P<0.001)$. Finally, the LOS of index ICU admission was longer in the three event groups than in the no event group $(P<0.001)$. Although SOFA scores at index ICU discharge were higher in the three event groups than in the no event group $(P<0.001)$, there were no significant differences in the difference values of SOFA between admission and discharge across the four groups $(P=0.540)$.

\section{Index ICU discharge date}

Vital signs and laboratory findings on the day of index ICU discharge are shown in Table 2. Heart rate was higher in the early (median 101/min), intermediate (median 99/min), and late event groups (median 97/min) than in the no event group (median 91/min; $P<0.001$ ). Patients with a lower GCS were more commonly identified in the early event group than in the no event group $(P=0.001)$; however, there were no differences between the other event groups and the no event group. Of 657 patients that underwent arterial blood gas analyses, there were no significant differences across the groups. There were statistically significant differences in the laboratory findings of platelet counts, total bilirubin, albumin, urea nitrogen, lactic acid, and N-terminal pro-brain natriuretic peptide (NT-proBNP); however, there were missing values for some variables.

\section{ICU readmission or unexpected ward death and final outcomes}

Patient characteristics of the event groups are shown in Table 3. Unexpected ward death was more frequent in the intermediate (12.5\%) and late event groups (37.2\%) than in the early event group $(2.4 \%, P<0.001)$. There were no differences in reason for ICU readmission across the event groups. Of 178 patients readmitted to the ICU, readmissions for the same reason as index admissions were found in 98 (55.1\%) cases. There were no differences on SAPS 3 and SOFA scores at ICU readmission across the event groups. In addition, there was no difference in the mortality of ICU readmission across the event groups (early $v s$. intermediate $v s$. late; $26.8 \%$ vs. $32.1 \%$ vs. $30.9 \% ; P=0.845$ ). However, hospital mortality was higher in the late event group than in the early event group (early $v s$. intermediate $v s$. late; $54.8 \% v s .65 .6 \% v s .76 .0 \%$; $P=0.026)$. 
Table 2. Vital signs, Glasgow coma scale, and laboratory findings on the day of index ICU discharge.

\begin{tabular}{|c|c|c|c|c|c|c|}
\hline Variables & $\begin{array}{l}\text { Total } \\
(\mathrm{N}=691)\end{array}$ & $\begin{array}{l}\text { No event group* } \\
(\mathrm{n}=456)\end{array}$ & $\begin{array}{l}\text { Early event group* } \\
(\mathrm{n}=42)\end{array}$ & $\begin{array}{l}\text { Intermediate event group* } \\
(\mathrm{n}=64)\end{array}$ & $\begin{array}{l}\text { Late event group* } \\
(\mathrm{n}=129)\end{array}$ & $P$ \\
\hline \multicolumn{7}{|l|}{ Vital sign } \\
\hline Mean ABP, mmHg & $87(76-97)$ & $83(75-95)$ & $91(80-100)$ & $92(83-103)$ & $89(77-97)$ & $0.001^{\mathrm{b}}$ \\
\hline Heart rate, /min & $92(79-105)$ & $91(78-102)$ & $101(88-118)$ & $99(83-112)$ & $97(83-108)$ & $<0.001^{\mathrm{a}, \mathrm{b}, \mathrm{c}}$ \\
\hline Respiratory rate, /min & $20(16-24)$ & $19(16-23)$ & $22(17-27)$ & $21(16-24)$ & $21(17-25)$ & 0.047 \\
\hline Body temperature, ${ }^{\circ} \mathrm{C}$ & $\begin{array}{l}36.6(36.3- \\
37.1)\end{array}$ & $36.6(36.3-37.1)$ & $36.8(36.5-37.2)$ & $36.7(36.3-37.2)$ & $36.6(36.2-36.9)$ & 0.161 \\
\hline Glasgow coma scale & & & & & & $0.001^{\mathrm{a}}$ \\
\hline 15 & $515(74.5)$ & $350(76.8)$ & $19(45.2)$ & $47(73.4)$ & $99(76.7)$ & \\
\hline $11-14$ & $143(20.7)$ & $87(19.1)$ & $16(38.1)$ & $15(23.4)$ & $25(19.4)$ & \\
\hline$\leq 10$ & $33(4.8)$ & $19(4.2)$ & $7(16.7)$ & $2(3.1)$ & $5(3.9)$ & \\
\hline \multicolumn{7}{|l|}{ Arterial blood gas $(n=657)$} \\
\hline $\mathrm{PaO}_{2} / \mathrm{FiO}_{2}$ & $405(355-479)$ & $403(356-480)$ & $419(345-510)$ & $407(357-443)$ & $418(352-507)$ & 0.819 \\
\hline $\mathrm{pH}$ & $\begin{array}{l}7.47(7.44- \\
7.50)\end{array}$ & $7.47(7.44-7.50)$ & $7.46(7.41-7.50)$ & $7.48(7.44-7.50)$ & $7.47(7.44-7.50)$ & 0.671 \\
\hline $\mathrm{PaCO}_{2}, \mathrm{mmHg}$ & $\begin{array}{l}33.7(30.4- \\
38.5)\end{array}$ & $33.5(30.2-37.9)$ & $35.6(32.2-40.8)$ & $33.8(31.7-38.8)$ & $33.8(30.0-39.8)$ & 0.058 \\
\hline $\mathrm{HCO}_{3}, \mathrm{mmol} / \mathrm{L}$ & $\begin{array}{l}24.9(22.0- \\
28.1)\end{array}$ & $24.5(21.8-27.9)$ & $26.0(24.0-29.4)$ & $25.5(23.1-28.7)$ & $24.9(21.5-28.3)$ & 0.063 \\
\hline \multicolumn{7}{|l|}{ Laboratory finding } \\
\hline White blood cell, $\times 10^{3} / \mu \mathrm{L}$ & $\begin{array}{l}7.26(4.16- \\
11.55)\end{array}$ & $7.21(4.45-11.14)$ & $7.07(1.96-12.95)$ & $7.46(3.39-12.53)$ & $7.75(2.89-13.13)$ & 0.902 \\
\hline Hemoglobin, g/dL & $9.9(9.0-11.2)$ & $10.0(9.2-11.2)$ & $9.6(8.9-10.3)$ & $9.7(9.0-11.0)$ & $9.8(8.9-11.4)$ & 0.067 \\
\hline Platelets, $\times 10^{3} / \mu \mathrm{L}$ & $107(40-214)$ & $130(48-229)$ & $51(22-92)$ & $63(32-175)$ & $77(35-175)$ & $<0.001^{\mathrm{a}, \mathrm{b}, \mathrm{c}}$ \\
\hline Total bilirubin, mg/dL & $0.8(0.5-1.5)$ & $0.7(0.4-1.5)$ & $1.1(0.6-2.9)$ & $1.0(0.6-1.8)$ & $0.8(0.5-1.5)$ & $0.002^{\mathrm{a}, \mathrm{b}}$ \\
\hline Albumin, g/dL $(\mathrm{n}=620)$ & $2.9(2.6-3.2)$ & $2.9(2.6-3.3)$ & $2.8(2.5-3.0)$ & $2.8(2.5-3.1)$ & $2.8(2.5-3.1)$ & 0.036 \\
\hline Urea nitrogen, $\mathrm{mg} / \mathrm{dL}$ & $\begin{array}{l}20.4(13.0- \\
30.2)\end{array}$ & $17.9(12.6-27.2)$ & $24.1(12.1-34.0)$ & $27.3(17.7-40.2)$ & $22.7(15.7-33.2)$ & $<0.001^{\mathrm{b}, \mathrm{c}}$ \\
\hline Creatinine, $\mathrm{mg} / \mathrm{dL}$ & $0.7(0.5-1.0)$ & $0.7(0.5-1.0)$ & $0.6(0.5-1.0)$ & $0.8(0.5-1.2)$ & $0.7(0.5-1.2)$ & 0.657 \\
\hline $\begin{array}{l}\text { Sodium, } \mathrm{mmol} / \mathrm{L} \\
(\mathrm{n}=687)\end{array}$ & $138(134-141)$ & $138(134-141)$ & $139(135-143)$ & $137(135-142)$ & $137(134-140)$ & 0.282 \\
\hline $\begin{array}{l}\text { Lactic acid, } \mathrm{mmol} / \mathrm{L} \\
(\mathrm{n}=627)\end{array}$ & $\begin{array}{l}1.39(1.02- \\
1.97)\end{array}$ & $1.34(1.00-1.87)$ & $1.67(1.31-2.51)$ & $1.58(0.99-2.22)$ & $1.39(1.04-2.13)$ & $0.003^{\mathrm{a}}$ \\
\hline $\mathrm{CRP}, \mathrm{mg} / \mathrm{dL}(\mathrm{n}=545)$ & $6.6(3.2-12.8)$ & $6.6(3.1-13.0)$ & $6.9(2.6-12.1)$ & $7.1(2.3-15.2)$ & $6.1(3.8-11.3)$ & 0.955 \\
\hline $\begin{array}{l}\text { Procalcitonin, } \mathrm{ng} / \mathrm{mL} \\
(\mathrm{n}=402)\end{array}$ & $\begin{array}{l}0.94(0.23- \\
5.86)\end{array}$ & $0.96(0.22-6.37)$ & $1.57(0.40-7.73)$ & $0.82(0.22-2.97)$ & $0.78(0.26-4.61)$ & 0.692 \\
\hline $\begin{array}{l}\text { NT-proBNP, pg/mL } \\
(\mathrm{n}=332)\end{array}$ & $\begin{array}{l}1379(508- \\
5676)\end{array}$ & $1167(458-5077)$ & $4233(1336-17252)$ & $1927(263-4930)$ & $1620(699-7488)$ & $0.007^{\mathrm{a}}$ \\
\hline
\end{tabular}

Values are median with interquartile range or $\mathrm{n}(\%)$.

*No event group means patients who were discharged alive from the hospital without ICU readmission. Early event group means patients who were unexpectedly readmitted to the ICU or died at the ward within 2 days after index ICU discharge. Intermediate event group means patients who were unexpectedly readmitted to the ICU or died at the ward between 2 and 7 days after index ICU discharge. Late event group means patients who were unexpectedly readmitted to the ICU or died at the ward after 7 days of index ICU discharge.

${ }^{\mathrm{a}} P<0.05$ with Bonferroni correction between no event and early event groups.

${ }^{\mathrm{b}} P<0.05$ with Bonferroni correction between no event and intermediate event groups.

${ }^{c} P<0.05$ with Bonferroni correction between no event and late event groups.

ICU, intensive care unit; ABP, arterial blood pressor; CRP, C-reactive protein; NT-proBNP, N-terminal pro-brain natriuretic peptide.

https://doi.org/10.1371/journal.pone.0211240.t002 
Table 3. ICU readmission status and final outcomes.

\begin{tabular}{|c|c|c|c|c|c|}
\hline Variables & Total $(\mathrm{N}=235)$ & Early event group* $(n=42)$ & Intermediate event group* $(n=64)$ & Late event group* $(n=129)$ & $P$ \\
\hline $\begin{array}{l}\text { Death at general ward before } \\
\text { ICU readmission }\end{array}$ & $57(24.3)$ & $1(2.4)$ & $8(12.5)$ & $48(37.2)$ & $<0.001^{\mathrm{a}, \mathrm{b}, \mathrm{c}}$ \\
\hline \multicolumn{6}{|l|}{ Reason for ICU readmission } \\
\hline Respiratory failure & $74 / 178(41.6)$ & $18 / 41(43.9)$ & $24 / 56(42.9)$ & $32 / 81(39.5)$ & 0.873 \\
\hline Sepsis & $60 / 178(33.7)$ & $12 / 41(29.3)$ & $15 / 56(26.8)$ & $33 / 81(40.7)$ & 0.187 \\
\hline Cardiovascular & $12 / 178(6.7)$ & $4 / 41(9.8)$ & $4 / 56(7.1)$ & $4 / 81(4.9)$ & 0.590 \\
\hline Bleeding & $9 / 178(5.1)$ & $2 / 41(4.9)$ & $4 / 56(7.1)$ & $3 / 81(3.7)$ & 0.685 \\
\hline Neurologic disorder & $9 / 178(5.1)$ & $2 / 41(4.9)$ & $5 / 56(8.9)$ & $2 / 81(2.5)$ & 0.273 \\
\hline Others & $14 / 178(7.9)$ & $3 / 41(7.3)$ & $4 / 56(7.1)$ & $7 / 81(8.6)$ & 1.000 \\
\hline Same reason as index ICU & 98/178 (55.1) & $22 / 41(53.7)$ & $34 / 56(60.7)$ & $42 / 81(51.9)$ & 0.579 \\
\hline SAPS III at ICU readmission & $64(50-75)$ & $66(48-76)$ & $58(44-78)$ & $65(53-72)$ & 0.497 \\
\hline SOFA at ICU readmission & $8(5-12)$ & $9(5-12)$ & $7(5-12)$ & $8(5-11)$ & 0.615 \\
\hline Mortality of ICU readmission & $54 / 178(30.3)$ & $11 / 41(26.8)$ & $18 / 56(32.1)$ & $25 / 81(30.9)$ & 0.845 \\
\hline LOS of ICU readmission & $4(2-9)$ & $7(4-10)$ & $5(2-11)$ & $3(1-8)$ & $0.017^{\mathrm{b}}$ \\
\hline Hospital LOS $^{\mathrm{d}}$ & $39(24-77)$ & $36(20-66)$ & $34(21-63)$ & $49(28-84)$ & $0.015^{\mathrm{c}}$ \\
\hline Hospital mortality $^{\mathrm{e}}$ & $163(69.4)$ & $23(54.8)$ & $42(65.6)$ & $98(76.0)$ & $0.026^{\mathrm{c}}$ \\
\hline
\end{tabular}

Values are median with interquartile range or $\mathrm{n}(\%)$.

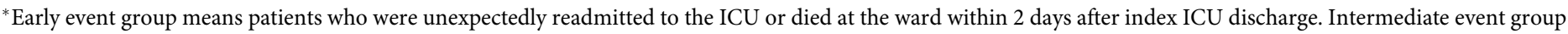

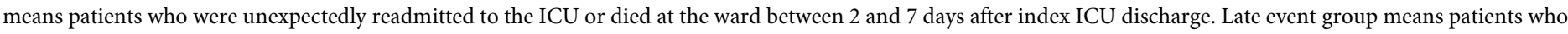
were unexpectedly readmitted to the ICU or died at the ward after 7 days of index ICU discharge.

${ }^{a} P<0.05$ with Bonferroni correction between early and intermediate event groups.

${ }^{\mathrm{b}} P<0.05$ with Bonferroni correction between early and late event groups.

${ }^{\mathrm{c}} P<0.05$ with Bonferroni correction between intermediate and late event groups.

${ }^{\mathrm{d}}$ Hospital LOS in no event group was a median of 17 days (IQR, 9-30 days).

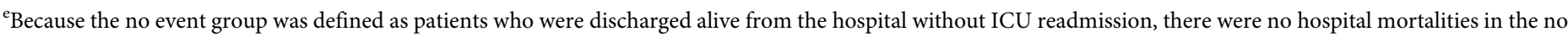
event group. Of a total of 691 patients, the hospital mortality was 163 (23.6\%).

ICU, intensive care unit; SAPS, Simplified Acute Physiology Score; SOFA, Sequential Organ Failure Assessment; LOS, length of stay; IQR, interquartile range.

\section{Independent variables associated with ICU readmission or unexpected ward death}

Multinomial logistic regression analysis to identify the independent variables associated with unplanned ICU readmission or unexpected ward death depending on the time and the index ICU discharge is shown in Table 4. Comorbidities such as chronic obstructive pulmonary disease (COPD) and asthma, mechanical ventilation during index ICU admission, lower GCS, and platelet count on the day of index ICU discharge were associated with early event group. Index ICU admission from the ER and higher urea nitrogen on the day of index ICU discharge were more frequent in the intermediate event group. The late event group was associated with comorbidity such as chronic heart disease and COPD/asthma, chemotherapy within 4 weeks before index ICU admission, index ICU admission from the ER, reasons for index ICU admission including sepsis and respiratory failure, a heart rate more than 100 beats/min, and higher blood urea nitrogen on the day of index ICU discharge. While different variables were associated with ICU readmission or ward death, none of the associated variables were statistically significant across the three event groups.

\section{Discussion}

In this study, we investigated the epidemiology, characteristics including disease-specific and treatment-related variables, and clinical outcomes of patients who experienced ICU 
Table 4. Multinomial logistic regression analysis of ICU readmission or ward death after ICU discharge ${ }^{\dagger}$.

\begin{tabular}{|c|c|c|c|c|c|c|c|c|}
\hline \multirow[t]{2}{*}{ Variables } & \multirow[t]{2}{*}{$\mathbf{K}^{2}$} & \multirow[t]{2}{*}{$\boldsymbol{P}$} & \multicolumn{2}{|l|}{ Early event group* } & \multicolumn{2}{|c|}{ Intermediate event group* } & \multicolumn{2}{|l|}{ Late event group* } \\
\hline & & & aOR $(95 \% \mathrm{CI})$ & $P$ & aOR $(95 \% \mathrm{CI})$ & $P$ & aOR $(95 \% \mathrm{CI})$ & $P$ \\
\hline Sex, male & 8.94 & 0.030 & $0.83(0.40-1.73)$ & 0.622 & $1.90(0.96-3.76)$ & 0.066 & $0.66(0.42-1.02)$ & 0.064 \\
\hline \multicolumn{9}{|l|}{ Comorbidity } \\
\hline Chronic heart disease & 11.60 & 0.009 & $1.14(0.41-3.12)$ & 0.803 & $0.36(0.11-1.24)$ & 0.105 & $2.12(1.17-3.83)$ & 0.013 \\
\hline Chronic liver disease & 7.81 & 0.050 & $0.24(0.05-1.19)$ & 0.081 & $0.24(0.05-1.08)$ & 0.062 & $0.81(0.38-1.74)$ & 0.587 \\
\hline COPD/asthma & 10.72 & 0.013 & $9.32(2.45-35.45)$ & 0.001 & $1.98(0.59-6.69)$ & 0.271 & $2.52(1.02-6.28)$ & 0.046 \\
\hline Chemotherapy within 4 weeks & 8.04 & 0.045 & $0.68(0.32-1.42)$ & 0.304 & $0.86(0.48-1.55)$ & 0.617 & $0.52(0.33-0.83)$ & 0.006 \\
\hline Source of index ICU admission: ER & 15.97 & 0.001 & $1.30(0.64-2.60)$ & 0.468 & $2.05(1.15-3.67)$ & 0.015 & $2.20(1.41-3.42)$ & $<0.001$ \\
\hline \multicolumn{9}{|l|}{ Reason for index ICU admission } \\
\hline Sepsis & 8.77 & 0.033 & $1.27(0.52-3.12)$ & 0.598 & $0.68(0.32-1.45)$ & 0.315 & $2.15(1.18-3.91)$ & 0.012 \\
\hline Respiratory failure & 11.05 & 0.011 & $0.68(0.26-1.84)$ & 0.451 & $1.01(0.48-2.11)$ & 0.981 & $2.52(1.38-4.63)$ & 0.003 \\
\hline $\begin{array}{l}\text { Organ support during index ICU } \\
\text { admission: mechanical ventilation }\end{array}$ & 10.91 & 0.012 & $3.30(1.55-7.00)$ & 0.002 & $1.52(0.82-2.83)$ & 0.187 & $1.32(0.82-2.13)$ & 0.251 \\
\hline \multicolumn{9}{|l|}{ On the day of index ICU discharge } \\
\hline Heart rate $>100 / \mathrm{min}$ & 15.77 & 0.001 & $2.63(1.31-5.26)$ & 0.007 & $1.73(0.98-3.06)$ & 0.060 & $2.02(1.30-3.14)$ & 0.002 \\
\hline GCS & & & & & & & & \\
\hline 15 & 18.12 & 0.006 & 1.00 (reference) & & 1.00 (reference) & & 1.00 (reference) & \\
\hline $11-14$ & & & $2.64(1.22-5.73)$ & 0.014 & $0.96(0.48-1.91)$ & 0.908 & $0.69(0.40-1.20)$ & 0.186 \\
\hline$\leq 10$ & & & $6.92(2.22-21.55)$ & 0.001 & $0.54(0.11-2.63)$ & 0.446 & $0.74(0.25-2.18)$ & 0.589 \\
\hline \multicolumn{9}{|l|}{ Platelet } \\
\hline \begin{tabular}{l|l} 
& $>100, \times 10^{3} / \mu \mathrm{L}$
\end{tabular} & 19.48 & 0.003 & 1.00 (reference) & & 1.00 (reference) & & 1.00 (reference) & \\
\hline$>50$ but $\leq 100, \times 10^{3} / \mu \mathrm{L}$ & & & $5.63(1.96-16.14)$ & 0.001 & $0.93(0.36-2.41)$ & 0.875 & $1.73(0.93-3.22)$ & 0.081 \\
\hline$\leq 50, \times 10^{3} / \mu \mathrm{L}$ & & & $5.95(2.19-16.19)$ & $<0.001$ & $1.68(0.83-3.42)$ & 0.150 & $1.49(0.86-2.58)$ & 0.155 \\
\hline \multicolumn{9}{|l|}{ Urea nitrogen } \\
\hline$\leq 20 \mathrm{mg} / \mathrm{dL}$ & 18.42 & 0.005 & 1.00 (reference) & & 1.00 (reference) & & 1.00 (reference) & \\
\hline$>20$ but $\leq 40 \mathrm{mg} / \mathrm{dL}$ & & & $0.72(0.32-1.60)$ & 0.413 & $2.33(1.21-4.45)$ & 0.011 & $1.82(1.13-2.93)$ & 0.015 \\
\hline$>40 \mathrm{mg} / \mathrm{dL}$ & & & $0.65(0.24-1.77)$ & 0.398 & $3.51(1.55-7.97)$ & 0.003 & $1.88(0.99-3.58)$ & 0.056 \\
\hline
\end{tabular}

${ }^{\dagger}$ No event group was used as the reference category.

${ }^{*}$ No event group means patients who were discharged alive from the hospital without ICU readmission. Early event group means patients who were unexpectedly readmitted to the ICU or died at the ward within 2 days after index ICU discharge. Intermediate event group means patients who were unexpectedly readmitted to the ICU or died at the ward between 2 and 7 days after index ICU discharge. Late event group means patients who were unexpectedly readmitted to the ICU or died at the ward after 7 days of index ICU discharge.

This multinomial logistic regression analysis model had the following Pearson goodness-of-fit test result: $\chi^{2}=2036.173(P=0.408)$. ICU, intensive care unit; aOR, adjusted odds ratio; $\mathrm{CI}$, confidence interval; COPD, chronic obstructive pulmonary disease; ER, emergency room; GCS, Glasgow coma scale.

https://doi.org/10.1371/journal.pone.0211240.t004

readmission or death in the ward based on the time interval between discharge and readmission or unexpected ward death and compared them with those of patients who were discharged from the hospital alive without ICU readmission during their same hospitalization.

Overall, $34.0 \%$ of patients in our study were readmitted to the ICU $(25.8 \%)$ or died in the general ward (8.2\%) during hospitalization, and $6.1 \%, 9.2 \%$, and $18.7 \%$ of readmission or death in the ward occurred within 2 days, 2-7 days, and 7 days after ICU discharge, respectively. Sepsis and respiratory failure were common causes and accounted for $60-80 \%$ of ICU admission, regardless of whether it was the index admission or readmission and when the event occurred. Recent studies of the general population demonstrated that $3-17 \%$ of patients discharged from a mixed medical-surgical or medical ICU were readmitted to the ICU during the same hospitalization $[15,19,20]$. Although there is currently limited data on critically ill cancer patients, ICU readmission rates in this population are reported to be about 8-9\% 
[21,22]. Readmission to the ICU occurred at a relatively high rate in our study compared to the incidence reported in previous studies. This readmission rate could have been impacted by several factors. Although we excluded patients who decided not to be readmitted to the ICU, the severity of illness of our cohort was much higher than in previous studies [14,15,21,23]. The severity of illness at the time of ICU admission, which is assessed by various scoring systems, is known to be an independent risk factor for ICU readmission [14,19,24]. In addition, cancer is a comorbidity that can increase readmission rates [19,25].

Various patient-related factors such as age, male gender, comorbid conditions, admission diagnosis, use of organ support treatment during ICU stay, and ICU LOS [5,14,16,17,21,23,26] and organizational factors such as lack of ICU beds and out-of-hours discharge from the ICU have been identified as variables associated with increased ICU readmission or unexpected ward death in previous studies $[17,18,23,27]$. However, previous reports had various criteria for the timing of ICU readmission (within 24 hours [18], within 48 hours [14,16], within 7 days [17], and no limitation $[5,21,23,27])$. In addition, several reports excluded unexpected death on the ward in the analysis $[5,21,23,27]$. Although ICU readmission and unexpected death on the ward might have common risk factors, it might vary depending on the timing of ICU readmission and death on the ward after index ICU discharge. Therefore, our study identified variables that were related to readmission to the ICU or death on the ward within 2 days, between 2 and 7 days, and after 7 days following index ICU discharge. Organ dysfunction on the day of index ICU discharge (decreased mental status, tachycardia, and thrombocytopenia) and organ support treatment during index ICU stay (need for mechanical ventilation) mainly affected the early readmitted or unexpectedly died patients. Considering that the median SOFA score at the index ICU discharge in the early event group was higher than in other groups, ICU discharge before stabilization of acute illness could be related to early readmission or death on the ward. This is supported by the European Society of Intensive Care Medicine, indicating that the ICU readmission rate within 48 hours of ICU discharge is mainly explained by residual organ dysfunction/failure at ICU discharge [28].

Although specific reasons for index ICU admission including sepsis and respiratory failure was associated with late readmission or unexpected ward death, we hypothesized that the patient's medical condition after sepsis or respiratory failure, such as worsening pre-illness status, new impaired function, and frailty, might affect readmission or death more than incomplete recovery from acute illness $[29,30]$. As a result, an underlying medical condition such as chronic heart disease, COPD/asthma, and chemotherapy within 4 weeks influenced ICU readmission or death on the ward after 7 days of ICU discharge. Therefore, improving general conditions and managing chronic illness might be an effective way to prevent late readmission or unexpected death on the ward.

Previous studies demonstrated that patients with at least one ICU readmission had a higher mortality than patients who did not $[19,21]$. In our cohort, mortality rate at index ICU hospitalization was $23.6 \%$, and overall ICU mortality of patients readmitted to the ICU was $30.3 \%$. ICU mortalities among patients readmitted to the ICU within 2 days, between 2 and 7 days, and after 7 days following index ICU discharge were similar, but hospital mortality in the late event group was higher than others, and patients typically had a longer hospital stay. This is in agreement with previous studies showing that ICU readmission is associated with a longer hospital stay $[4,5]$.

This study provides information on factors associated with ICU readmission or unexpected ward death and clinical outcomes in critically ill cancer patients discharged alive from the ICU. Furthermore, we characterized cancer patients who were readmitted to the ICU or unexpectedly died on the ward based on the time interval between ICU discharge and the events as early, intermediate, and late. However, there were several limitations that should be 
considered. First, given the retrospective and observational nature of our study, there is a potential risk of selection bias and confounding variables influencing the significance of our findings. However, the data were prospectively collected from all of the critically ill cancer patients consecutively admitted to the medical ICU. In addition, our study was conducted at a single institution with a specialized ICU for critically ill cancer patients. Considering that rates of readmission to the ICU varied by geographic region, hospital, and type of ICU [31,32], the generalizability of our findings to other centers is limited. Second, some of the associated factors to the early events such as tachycardia and mental deterioration could indicate a premature ICU discharge, since there was no predefined objective criterion for ICU discharge. Third, we used the time interval from ICU discharge to ICU transfer in order to divide the group of patients readmitted to the ICU or unexpectedly died on the ward. Therefore, it is difficult to exclude the possibility that there is a gap between the time of decision to readmit to the ICU and the time transferred to the ICU due to delayed transfers due to lack of bed availability. Fourth, the information about limitation of care decision, which is a variable that may affect the mortality, collected only at the time of the discharge from ICU and changes in the decision were not followed during the rest of the hospitalization. In addition, we only focused on the patient-related factors in the analysis, without consideration of organizational factors relevant to readmission according to previous studies $[18,33]$.

\section{Conclusions}

In summary, about one-third of critically ill cancer patients were readmitted to the ICU or died on the ward after being discharged alive from the index ICU admission. Risk factors associated with ICU readmission and mortality were different across the time intervals between discharge and events. Therefore, it is necessary to carefully assess residual organ dysfunction at ICU discharge as well as the treatment of underlying disease after ICU discharge in order to reduce ICU readmission and hospital mortality in critically ill cancer patients.

\section{Author Contributions}

Conceptualization: Byeong-Ho Jeong, Kyeongman Jeon.

Data curation: Byeong-Ho Jeong, Soo Jin Na, Dae-Sang Lee.

Formal analysis: Byeong-Ho Jeong, Soo Jin Na, Kyeongman Jeon.

Funding acquisition: Kyeongman Jeon.

Methodology: Soo Jin Na, Dae-Sang Lee, Chi Ryang Chung, Gee Young Suh, Kyeongman Jeon.

Supervision: Chi Ryang Chung, Gee Young Suh, Kyeongman Jeon.

Writing - original draft: Byeong-Ho Jeong, Soo Jin $\mathrm{Na}$, Kyeongman Jeon.

Writing - review \& editing: Chi Ryang Chung, Gee Young Suh, Kyeongman Jeon.

\section{References}

1. van Vliet M, Verburg IW, van den Boogaard M, de Keizer NF, Peek N, Blijlevens NM, et al. Trends in admission prevalence, illness severity and survival of haematological patients treated in Dutch intensive care units. Intensive Care Med. 2014; 40:1275-84. https://doi.org/10.1007/s00134-014-3373-x PMID: 24972886

2. Soares M, Caruso P, Silva E, Teles JM, Lobo SM, Friedman G, et al. Characteristics and outcomes of patients with cancer requiring admission to intensive care units: a prospective multicenter study. Crit Care Med. 2010; 38:9-15. https://doi.org/10.1097/CCM.0b013e3181c0349e PMID: 19829101 
3. Azoulay E, Mokart D, Pene F, Lambert J, Kouatchet A, Mayaux J, et al. Outcomes of critically ill patients with hematologic malignancies: prospective multicenter data from France and Belgium-a groupe de recherche respiratoire en reanimation onco-hematologique study. J Clin Oncol. 2013; 31:2810-8. https://doi.org/10.1200/JCO.2012.47.2365 PMID: 23752112

4. Kramer AA, Higgins TL, Zimmerman JE. The association between ICU readmission rate and patient outcomes. Crit Care Med. 2013; 41:24-33. https://doi.org/10.1097/CCM.0b013e3182657b8a PMID: 23128381

5. Kramer AA, Higgins TL, Zimmerman JE. Intensive care unit readmissions in U.S. hospitals: patient characteristics, risk factors, and outcomes. Crit Care Med. 2012; 40:3-10. https://doi.org/10.1097/ CCM.0b013e31822d751e PMID: 21926603

6. Taccone FS, Artigas AA, Sprung CL, Moreno R, Sakr Y, Vincent JL. Characteristics and outcomes of cancer patients in European ICUs. Crit Care. 2009; 13:R15. https://doi.org/10.1186/cc7713 PMID: 19200368

7. Song JU, Suh GY, Park HY, Lim SY, Han SG, Kang YR, et al. Early intervention on the outcomes in critically ill cancer patients admitted to intensive care units. Intensive Care Med. 2012; 38:1505-13. https:// doi.org/10.1007/s00134-012-2594-0 PMID: 22592633

8. Nates JL, Nunnally M, Kleinpell R, Blosser S, Goldner J, Birriel B, et al. ICU Admission, Discharge, and Triage Guidelines: A Framework to Enhance Clinical Operations, Development of Institutional Policies, and Further Research. Crit Care Med. 2016; 44:1553-602. https://doi.org/10.1097/ccm. 0000000000001856 PMID: 27428118

9. Benoit DD, Depuydt PO, Vandewoude KH, Offner FC, Boterberg T, De Cock CA, et al. Outcome in severely ill patients with hematological malignancies who received intravenous chemotherapy in the intensive care unit. Intensive Care Med. 2006; 32:93-9. https://doi.org/10.1007/s00134-005-2836-5 PMID: 16308681

10. Darmon M, Thiery G, Ciroldi M, de Miranda S, Galicier L, Raffoux E, et al. Intensive care in patients with newly diagnosed malignancies and a need for cancer chemotherapy. Crit Care Med. 2005; 33:248893. PMID: 16276171

11. Lee DS, Suh GY, Ryu JA, Chung CR, Yang JH, Park CM, et al. Effect of Early Intervention on LongTerm Outcomes of Critically III Cancer Patients Admitted to ICUs. Crit Care Med. 2015; 43:1439-48. https://doi.org/10.1097/CCM.0000000000000989 PMID: 25803653

12. Yoo H, Suh GY, Jeong BH, Lim SY, Chung MP, Kwon OJ, et al. Etiologies, diagnostic strategies, and outcomes of diffuse pulmonary infiltrates causing acute respiratory failure in cancer patients: a retrospective observational study. Crit Care. 2013; 17:R150. https://doi.org/10.1186/cc12829 PMID: 23880212

13. Song JU, Suh GY, Chung MP, Kim H, Kwon OJ, Jung CW, et al. Risk factors to predict outcome in critically ill cancer patients receiving chemotherapy in the intensive care unit. Support Care Cancer. 2011; 19:491-5. https://doi.org/10.1007/s00520-010-0841-x PMID: 20229331

14. Rosa RG, Roehrig C, Oliveira RP, Maccari JG, Antonio AC, Castro Pde S, et al. Comparison of Unplanned Intensive Care Unit Readmission Scores: A Prospective Cohort Study. PLoS One. 2015; 10:e0143127. https://doi.org/10.1371/journal.pone.0143127 PMID: 26600463

15. Ouanes I, Schwebel C, Francais A, Bruel C, Philippart F, Vesin A, et al. A model to predict short-term death or readmission after intensive care unit discharge. J Crit Care. 2012; 27:422.e1-9. https://doi. org/10.1016/j.jcrc.2011.08.003 PMID: 22172798

16. Lee H, Lim CW, Hong HP, Ju JW, Jeon YT, Hwang JW, et al. Efficacy of the APACHE II score at ICU discharge in predicting post-ICU mortality and ICU readmission in critically ill surgical patients. Anaesth Intensive Care. 2015; 43:175-86. PMID: 25735682

17. Chrusch CA, Olafson KP, McMillan PM, Roberts DE, Gray PR. High occupancy increases the risk of early death or readmission after transfer from intensive care. Crit Care Med. 2009; 37:2753-8. https:// doi.org/10.1097/CCM.0b013e3181a57b0c PMID: 19707139

18. Town JA, Churpek MM, Yuen TC, Huber MT, Kress JP, Edelson DP. Relationship between ICU bed availability, ICU readmission, and cardiac arrest in the general wards. Crit Care Med. 2014; 42:203741. https://doi.org/10.1097/ccm.0000000000000401 PMID: 24776607

19. Ponzoni CR, Correa TD, Filho RR, Serpa Neto A, Assuncao MSC, Pardini A, et al. Readmission to the Intensive Care Unit: Incidence, Risk Factors, Resource Use, and Outcomes. A Retrospective Cohort Study. Ann Am Thorac Soc. 2017; 14:1312-9. https://doi.org/10.1513/AnnalsATS.201611-851OC PMID: 28530118

20. Ranzani OT, Prada LF, Zampieri FG, Battaini LC, Pinaffi JV, Setogute YC, et al. Failure to reduce Creactive protein levels more than $25 \%$ in the last 24 hours before intensive care unit discharge predicts higher in-hospital mortality: a cohort study. J Crit Care. 2012; 27:525.e9-15. https://doi.org/10.1016/j. jcrc.2011.10.013 PMID: 22227090 
21. Rodrigues CM, Pires EM, Feliciano JP, Vieira JM Jr., Taniguchi LU. Admission factors associated with intensive care unit readmission in critically ill oncohematological patients: a retrospective cohort study. Rev Bras Ter Intensiva. 2016; 28:33-9. https://doi.org/10.5935/0103-507x.20160011 PMID: 27096674

22. Song SW, Lee HS, Kim JH, Kim MS, Lee JM, Zo JI. Readmission to intensive care unit after initial recovery from major thoracic oncology surgery. Ann Thorac Surg. 2007; 84:1838-46; discussion -46. https:// doi.org/10.1016/j.athoracsur.2007.06.074 PMID: 18036895

23. Renton J, Pilcher DV, Santamaria JD, Stow P, Bailey M, Hart G, et al. Factors associated with increased risk of readmission to intensive care in Australia. Intensive Care Med. 2011; 37:1800-8. https://doi.org/ 10.1007/s00134-011-2318-x PMID: 21845504

24. Frost SA, Alexandrou E, Bogdanovski T, Salamonson Y, Davidson PM, Parr MJ, et al. Severity of illness and risk of readmission to intensive care: a meta-analysis. Resuscitation. 2009; 80:505-10. https://doi. org/10.1016/j.resuscitation.2009.02.015 PMID: 19342149

25. Brown SE, Ratcliffe SJ, Kahn JM, Halpern SD. The epidemiology of intensive care unit readmissions in the United States. Am J Respir Crit Care Med. 2012; 185:955-64. https://doi.org/10.1164/rccm. 201109-17200C PMID: 22281829

26. Rosenberg AL, Watts $\mathrm{C}$. Patients readmitted to ICUs*: a systematic review of risk factors and outcomes. Chest. 2000; 118:492-502. PMID: 10936146

27. Pilcher DV, Duke GJ, George C, Bailey MJ, Hart G. After-hours discharge from intensive care increases the risk of readmission and death. Anaesth Intensive Care. 2007; 35:477-85. PMID: 18020063

28. Rhodes A, Moreno RP, Azoulay E, Capuzzo M, Chiche JD, Eddleston J, et al. Prospectively defined indicators to improve the safety and quality of care for critically ill patients: a report from the Task Force on Safety and Quality of the European Society of Intensive Care Medicine (ESICM). Intensive Care Med. 2012; 38:598-605. https://doi.org/10.1007/s00134-011-2462-3 PMID: 22278594

29. Shankar-Hari M, Rubenfeld GD. Understanding Long-Term Outcomes Following Sepsis: Implications and Challenges. Curr Infect Dis Rep. 2016; 18:37. https://doi.org/10.1007/s11908-016-0544-7 PMID: 27709504

30. Baldwin MR, Reid MC, Westlake AA, Rowe JW, Granieri EC, Wunsch H, et al. The feasibility of measuring frailty to predict disability and mortality in older medical intensive care unit survivors. J Crit Care. 2014; 29:401-8. https://doi.org/10.1016/j.jcrc.2013.12.019 PMID: 24559575

31. Hosein FS, Roberts DJ, Turin TC, Zygun D, Ghali WA, Stelfox HT. A meta-analysis to derive literaturebased benchmarks for readmission and hospital mortality after patient discharge from intensive care. Crit Care. 2014; 18:715. https://doi.org/10.1186/s13054-014-0715-6 PMID: 25551448

32. van Sluisveld N, Bakhshi-Raiez F, de Keizer N, Holman R, Wester G, Wollersheim H, et al. Variation in rates of ICU readmissions and post-ICU in-hospital mortality and their association with ICU discharge practices. BMC Health Serv Res. 2017; 17:281. https://doi.org/10.1186/s12913-017-2234-z PMID: 28416016

33. Gantner D, Farley K, Bailey M, Huckson S, Hicks P, Pilcher D. Mortality related to after-hours discharge from intensive care in Australia and New Zealand, 2005-2012. Intensive Care Med. 2014; 40:1528-35. https://doi.org/10.1007/s00134-014-3438-x PMID: 25118868 